\title{
Dielectric relaxations of confined water in porous silica ceramics
}

\author{
F GRAJA $^{1,2}$, A TRIKI ${ }^{3, *}$, MED TABELLOUT ${ }^{4}$, MED GARGOURI ${ }^{1}$ and C DEPOLLIER ${ }^{2}$ \\ ${ }^{1}$ Laboratory of Spectroscopy, Characterization and Optics of Materials, University of Sfax, \\ 3018 Sfax, Tunisia \\ ${ }^{2}$ Acoustic Laboratory, Maine University, 72085 Le Mans, France \\ ${ }^{3}$ Laboratory of Composite Materials, Ceramics and Polymers, University of Sfax, 3018 Sfax, Tunisia \\ ${ }^{4}$ Laboratory IMMM-PEC, UMRCNRS 628, Maine University, 72085 Le Mans, France \\ *Author for correspondence (trikilamacop@yahoo.fr)
}

MS received 17 October 2016; accepted 6 April 2017; published online 6 December 2017

\begin{abstract}
In this study, dielectric properties of water confined in porous silica ceramics were investigated. Two porous ceramics were characterized in the frequency range $10^{-1}$ to $10^{7} \mathrm{~Hz}$ and temperature interval from -100 to $200^{\circ} \mathrm{C}$. While the first sample was a ceramic with opened lateral pores, the second one was a ceramic with sealed lateral pores. In both ceramics, three dielectric processes were identified. The first, which appeared at lower temperatures, was attributed to the reorientation of water molecules in ice-like water cluster structures. The second is the relaxation observed over an intermediate temperature range, associated with the kinetic transition due to water molecule reorientation near a defect. At higher temperatures, the third was relaxation identified as the Maxwell-Wagner-Sillars polarization process due to the trapping of free charge carriers at the interface of the porous media. The first and second dielectric relaxations were analysed to prove the effect of the lateral surface state of the sample on water-inner surfaces of the porous media interaction. These analyses revealed a great similarity in the ice-like structure for both ceramics. However, the lateral surface state of the sample might enhance the dielectric strength of the first relaxation when lateral pores are sealed. Furthermore, it might improve the water-inner surfaces interaction when lateral pores are opened.
\end{abstract}

Keywords. Confined water; porous silica; dielectric relaxation; ceramics.

\section{Introduction}

Extensive studies have been undertaken on porous materials to understand the influence of their morphology on their dielectric properties better [1-5]. Among these materials, porous glasses show interesting properties, allowing their use in various applications [6-8]. Such materials can be considered as a matrix with a rigid sponge-like $\mathrm{SiO}_{2}$ framework that can be filled with different materials $[9,10]$. The dielectric properties of fillers confined in porous media were studied using various theoretical approaches and dielectric experimental techniques [2,3,9-12]. Water, which is the most used filler in such complex systems, can play a critical role in biological systems. In the case of porous silica media, water can easily be adsorbed and desorbed from silica surfaces. The dielectric studies of confined water in these media revealed the effect of pore size and chemical treatment on their structure and dynamics $[11,12]$.

Authors of this paper are interested in a porous medium that may occur in the bone of human body as a result of osteoporosis diseases. For this purpose, porous silica ceramics, which represent this structure, have been chosen. Dielectric measurements were carried out on these ceramics filled with water. The present study takes into account the lateral surface state of the sample by visualizing the existence or not of the outer wall of the bone, which represents the sealed lateral pores. The main purpose of this research work is to confirm the effect of these conditions on the water-inner surfaces of the porous media interaction.

\section{Experimental}

\subsection{Materials}

The porous silica ceramics used in this study were based on a commercially available Filtros ${ }^{\circledR}$, whose Trade Name is QF20 silica supplied by Ferro Corporation [13]. The physical properties of these ceramic materials were given in our previous study [14]. The analysed sample was cut using a diamond saw and the obtained thickness was about $2.78 \pm 0.05 \mathrm{~mm}$. Then, the sample was exposed to ultrasonic cleaning for $15 \mathrm{~min}$ in a water-acetone solution and then allowed to dry in a furnace at $100^{\circ} \mathrm{C}$ for $24 \mathrm{~h}$. Next, the sample was kept in a desiccator for $24 \mathrm{~h}$ to reach the ambient temperature. 


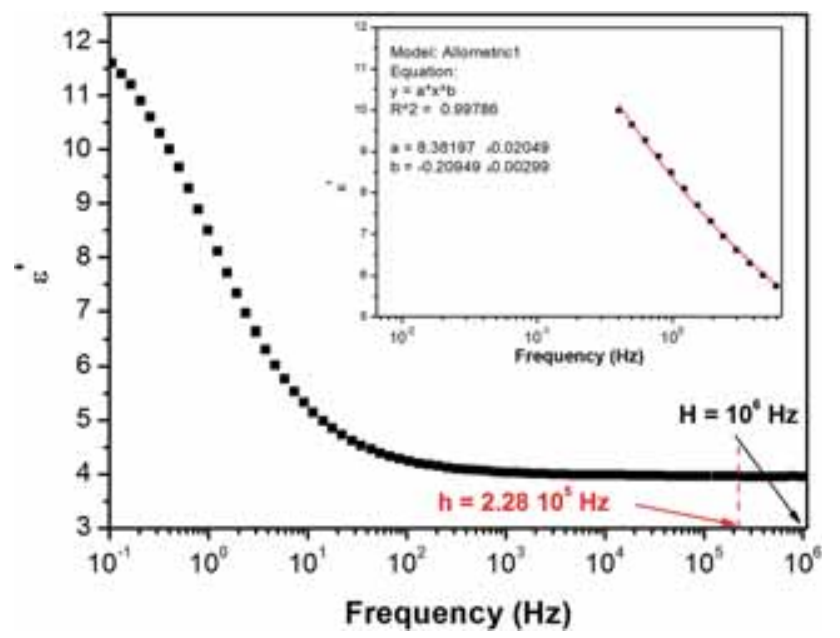

Figure 1. Variation of the dielectric permittivity as a function of frequency at the ambient for CWOLP. The inset shows the fitting of the dielectric permittivity decrease with the increase in frequency according to the following law: $\varepsilon^{\prime}(f) \approx f^{b}$.

\subsection{Characterization}

Dielectric measurements were realized in three different steps. In the first step, the dielectric measurements were performed at the ambient on a dry sample with opened lateral pores and in the frequency range $0.1 \mathrm{~Hz}-10 \mathrm{MHz}$. However, in the second and third steps, dielectric measurements were conducted in the temperature range -100 to $200^{\circ} \mathrm{C}$ in the increments of $10^{\circ} \mathrm{C}$ and in the frequency range $0.1 \mathrm{~Hz}-10$ $\mathrm{MHz}$. In the isothermal runs and the second step, the ceramic lateral pores were opened. The ceramic with opened lateral pores (CWOLP) was immersed in water for $15 \mathrm{~min}$ before its dielectric characterization. In the third step, the ceramic lateral pores were painted with a thin layer of Jeltargent, a conducting material allowing the obtention of sealed pores condition. In this condition, the immersion time of the ceramic with sealed lateral pores (CWSLP) in water was longer than that carried out in the second step. Its duration was about $14 \mathrm{~h}$. The relative water content in the dry sample was determined by weighing the ceramics prior to and immediately after the dielectric
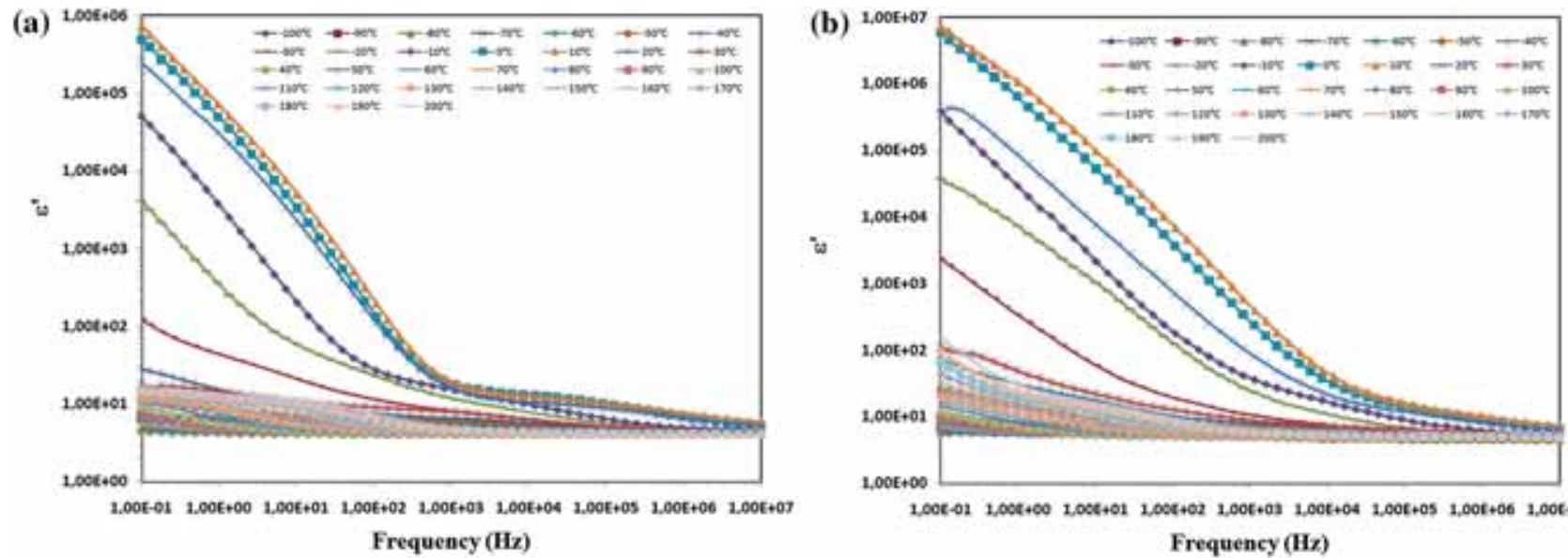

Frequency $(\mathrm{Hz})$

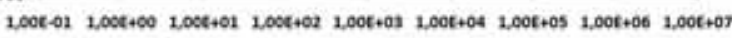
Frequency $(\mathbf{H z})$
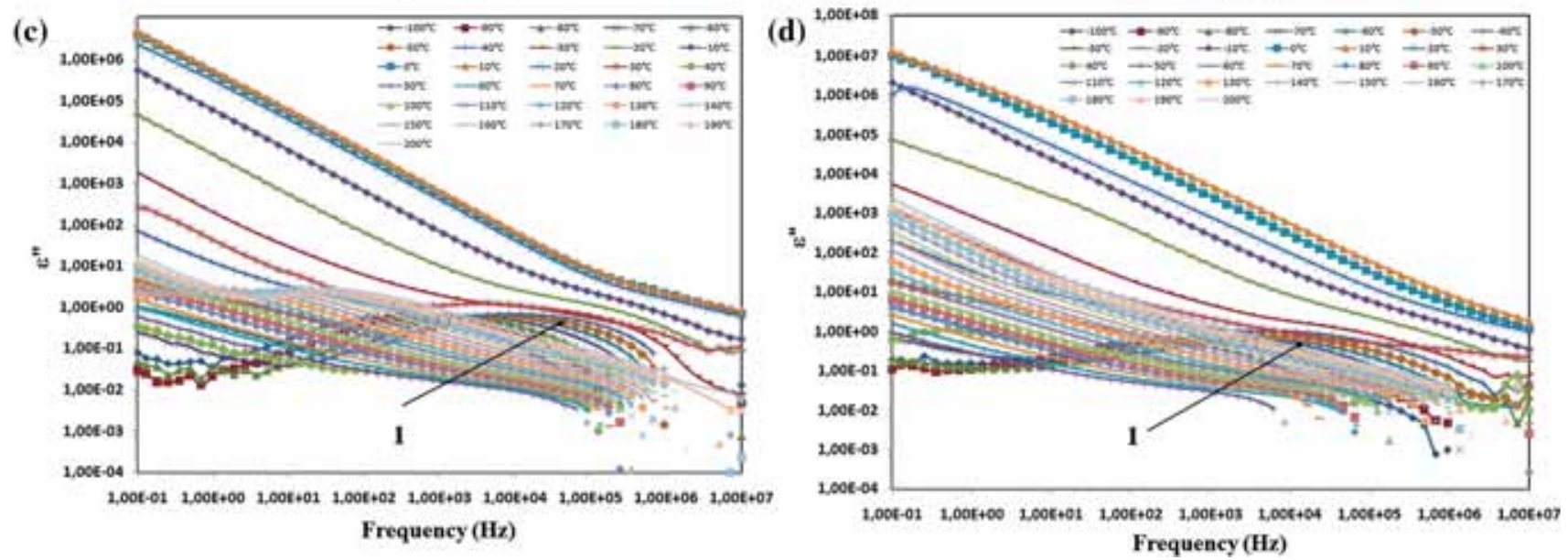

Figure 2. Isothermal runs of the dielectric permittivity $\left(\varepsilon^{\prime}\right)$ and of the loss factor $\left(\varepsilon^{\prime \prime}\right) v s$. frequency for (a, c) CWOLP and (b, d) CWSLP, respectively. 
measurements. The obtained humidity parameters were about 8.7 and $7.5 \%$ for CWOLP and CWSLP, respectively.

\section{Results and discussion}

\subsection{Determination of the porosity}

Figure 1 presents a typical power-law behaviour of the dielectric permittivity $v s$. frequency at the ambient for CWOLP. A similar behaviour was observed for other ceramic materials and disordered materials [15]. Such a behaviour is associated with processes that occur over fractal structures [16]. The use of fractal description allows relating the fractal dimension of the structure to the porosity [17]. The porosity can be written as follows [18]:

$$
\phi=\left(\frac{h}{H}\right)^{3-D_{\mathrm{f}}}
$$

where $h$ and $H$ are the lower and upper limits of similarity, respectively, and $D_{\mathrm{f}}$ is the fractal dimension of the object.

The inset of figure 1 depicts the fitting of the dielectric permittivity decrease with the increase of frequency according to the following law [17]:

$$
\varepsilon^{\prime}(f) \approx f^{\mathrm{b}}
$$

where the exponent $b$ is related to the fractal dimension by the relation $\left(D_{\mathrm{f}}-3\right) / 2$. It ranges between -1 and 0 . The obtained
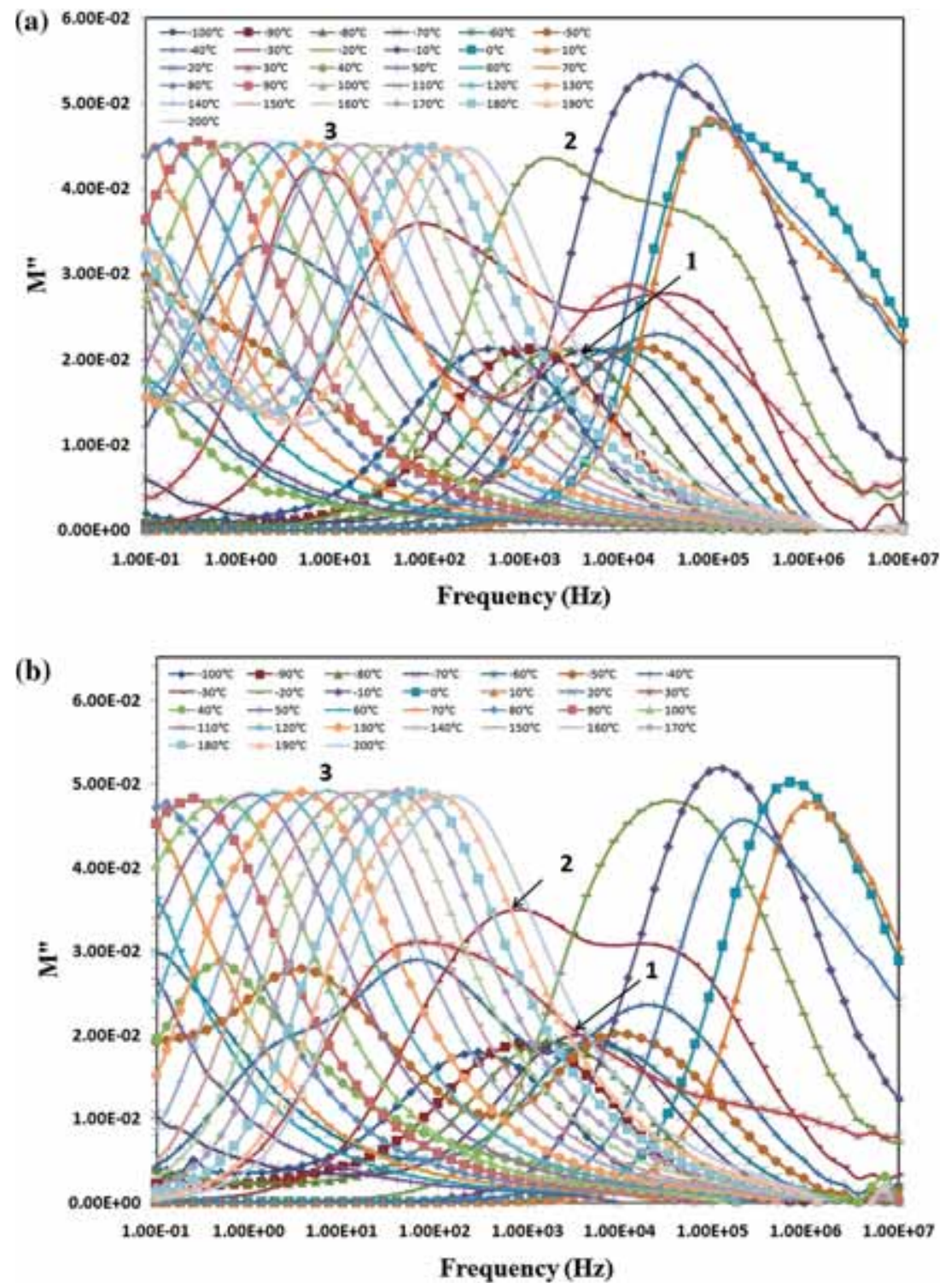

Figure 3. Frequency dependence of $M^{\prime \prime}$ for (a) CWOLP and (b) CWSLP. 
value is presented in the inset of figure 1. Consequently, the porosity $\phi$ determined by the dielectric permittivity measurements is about $53.9 \%$ for CWOLP. This value is in agreement with that given in our previous work [14].

\subsection{Dielectric relaxations analyses}

Comparative plots of the frequency dependence of the dielectric permittivity $\left(\varepsilon^{\prime}\right)$ and the loss factor $\left(\varepsilon^{\prime \prime}\right)$ in CWOLP and CWSLP for different temperatures from -100 to $200^{\circ} \mathrm{C}$ in the increments of $10^{\circ} \mathrm{C}$ are shown in figure $2 \mathrm{a}-\mathrm{d}$. An overall decrease in $\varepsilon^{\prime}$ with the increase in frequency and an increase in intensity with temperature at low frequencies were observed. The dispersion shown at low frequencies was more noticeable in the temperature range -30 to $20^{\circ} \mathrm{C}$. Accordingly, the loss factor in this temperature range exhibits a dc conductivity effect. This dielectric permittivity enhancement could be attributed to the kinetic transition due to water molecule reorientation in the vicinity of a defect [19]. Such a behaviour was also obtained for porous rocks ceramics. It is associated with the water bound in the system [17]. Besides, the loss factor, $\varepsilon^{\prime \prime}$, displayed the presence of only one dielectric relaxation at lower temperatures for both samples. This relaxation originates from the reorientation of water molecules in ice-like water cluster structures.

To minimize the effect of the dc conductivity, the formalism of the 'electric modulus' or 'inverse complex permittivity' is introduced. This electric modulus has recently been adapted for the description of dielectric processes occurring in systems with ionic conductivity [20]. The electric modulus, $M^{*}$, is defined by the following relationship [21]:

$$
M^{*}=\frac{1}{\varepsilon^{*}}=\frac{1}{\varepsilon^{\prime}}=\frac{\varepsilon^{\prime}}{\varepsilon^{\prime 2} \varepsilon^{\prime \prime 2}}+\mathrm{j} \frac{\varepsilon^{\prime \prime}}{\varepsilon^{\prime 2} \varepsilon^{\prime 2}}=M^{\prime}+\mathrm{j} M^{\prime \prime},
$$

where $M^{\prime}$ and $M^{\prime \prime}$ are the real and imaginary parts of electric modulus, respectively. An advantage of using the electric modulus to interpret bulk relaxation properties is that the variations in the large values of real part of permittivity and the loss factor at low frequencies are minimized. In this way, common difficulties of electrode nature and contact, space charge injection phenomena and absorbed impurity conduction effects, which appear to obscure relaxation in the permittivity representation, can be resolved or even ignored [22].

On transforming the loss factor values into the electric modulus formalism, figure $3 \mathrm{a}$ and $\mathrm{b}$ is obtained, giving the imaginary part, $M^{\prime \prime}$, of the electric modulus $v s$. frequency for CWOLP and CWSLP. The variation of the imaginary part $M^{\prime \prime}$ with temperature shows the presence of a series of three distinct relaxations as the CWOLP and CWSLP are heated over the temperature range -100 to $200^{\circ} \mathrm{C}$.

The first relaxation process, which is observed in the lowtemperature region from -100 to $-20^{\circ} \mathrm{C}$ for CWOLP and from -100 to $-30^{\circ} \mathrm{C}$ for CWSLP, is due to the reorientation of water molecules in ice-like water cluster structures.
The second relaxation process occurred in the temperature range -40 to $30^{\circ} \mathrm{C}$ for the CWOLP and -50 to $40^{\circ} \mathrm{C}$ for CWSLP, which is attributed to the kinetic transition due to water molecule reorientation in the vicinity of a defect [19].

The last relaxation process appeared in the high temperature region, above $70^{\circ} \mathrm{C}$ for CWOLP and above $80^{\circ} \mathrm{C}$ for CWSLP, which is associated with the Maxwell-WagnerSillars polarization process as a result of the trapping of free charge carriers at the interface of the porous media.

In this analytical dielectric study, the focus was on the interaction of water with the porous ceramic inner structure. For this purpose, quantitative dielectric analyses of the first and second relaxations were investigated.

The temperature dependences of the relaxation times of the first dielectric process for the CWOLP and CWSLP reveal Arrhenius behaviour, as illustrated in figure 4 . The mean values of activated energy, $E_{\mathrm{a} 1}$, related to the first relaxation are 22 and $22.73 \mathrm{~kJ} \mathrm{~mol}^{-1}$ for CWOLP and CWSLP, respectively (table 1 ). These values are significantly smaller than that for bulk ice $\left(\sim 60 \mathrm{~kJ} \mathrm{~mol}^{-1}\right)$ [23]. The decrease in $E_{\mathrm{a} 1}$ could be attributed to a less rigid ice-like structure in comparison with the bulk ice. Hence, the internal structure of porous media facilitates the reorientation of water molecules in icelike structure due to the presence of structural defects [24]. As a result, the relaxation times of the first relaxation illustrated in table 1 for CWOLP and CWSLP are smaller than that for bulk ice $\left(\sim 10^{-5} \mathrm{~s}\right)$. Taking into account the lateral surface state of the sample, it can be noted that this condition does

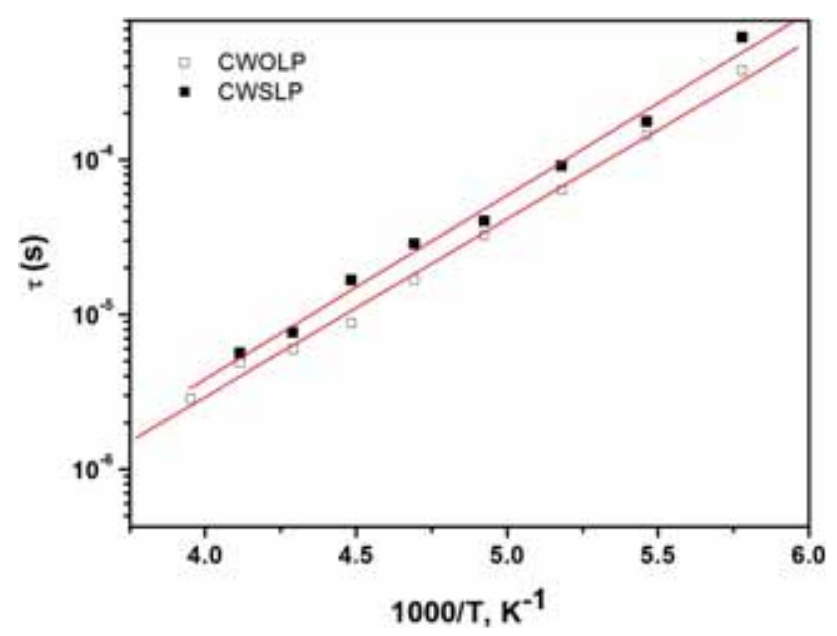

Figure 4. Arrhenius plots of the $\tau$ values vs. the reciprocal temperature for CWOLP and CWSLP.

Table 1. Activation energies $E_{\mathrm{a} 1}$ and relaxation times $\tau_{01}$ of the first dielectric relaxation for the CWOLP and CWSLP.

\begin{tabular}{lccc} 
Samples & Relaxation & $E_{\mathrm{a} 1}\left(\mathrm{~kJ} \mathrm{~mol}^{-1}\right)$ & $\tau_{01}(\mathrm{~s})$ \\
\hline CWOLP & 1 & 22 & $7.42 \times 10^{-11}$ \\
CWSLP & 1 & 22.73 & $6.91 \times 10^{-11}$ \\
\hline
\end{tabular}



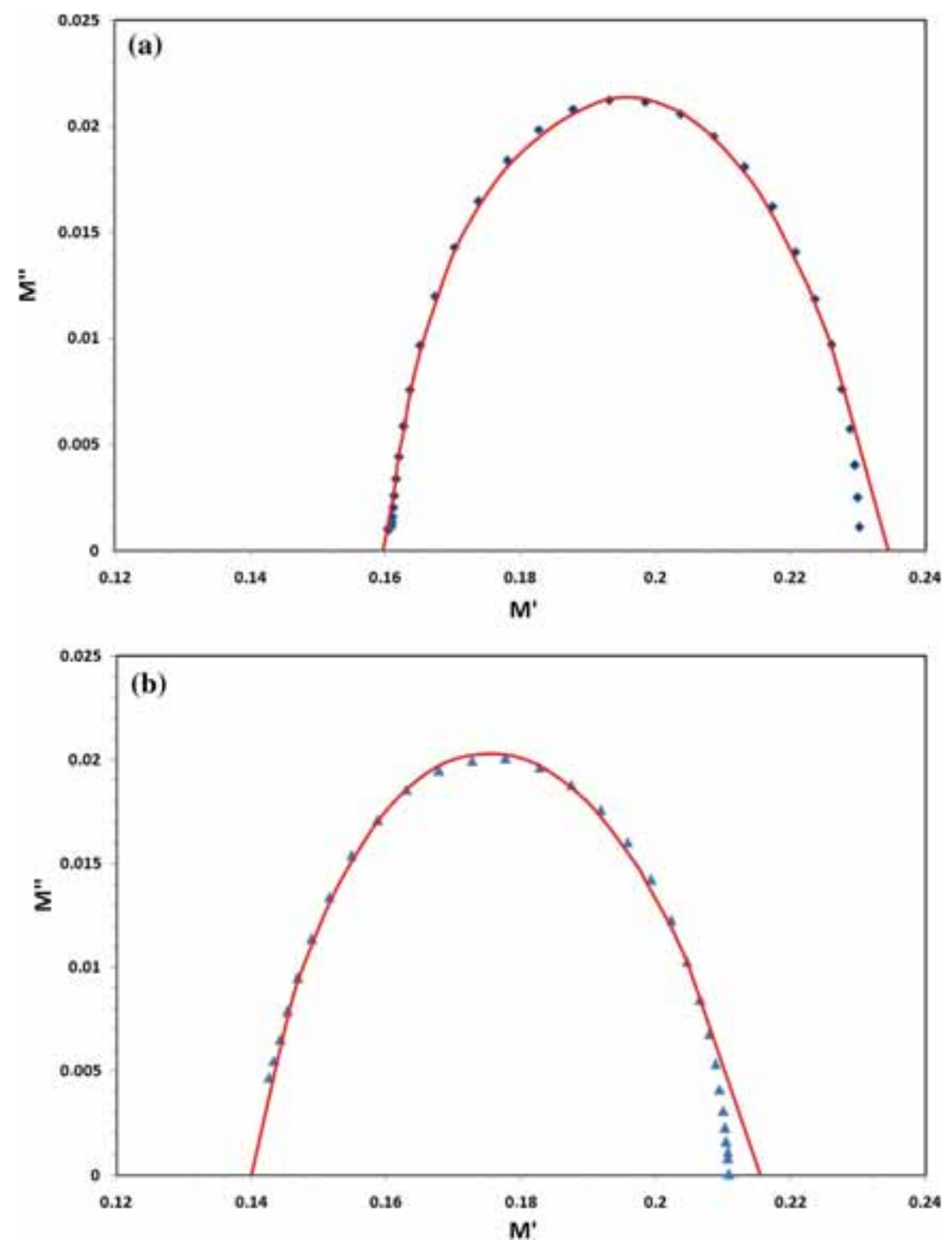

Figure 5. Argand's plots of $M^{*}$ for (a) CWOLP and (b) CWSLP at $-70^{\circ} \mathrm{C}$. Full lines correspond to the best fit according to equation (4).

not affect the reorientation of water molecules in the ice-like structure. This explains why the activation energies $E_{\mathrm{a} 1}$ for CWOLP and CWSLP are similar.

The Argand representation was used to analyse the nature of the first relaxation. Figure $5 \mathrm{a}$ and $\mathrm{b}$ presents an illustration of Cole-Cole plots at $-70^{\circ} \mathrm{C}$ for CWOLP and CWSLP, respectively.

It has been well established that the response of every relaxation mechanism can be represented very precisely by a model function with four parameters at the most. Among others, this includes the following function $[25,26]$ :

$$
M^{*}=M_{\infty}+\frac{M_{\mathrm{s}}-M_{\infty}}{\left(1+(\mathrm{j} \omega \tau)^{1-\alpha}\right)^{\beta}} .
$$

This function, which was introduced by Havriliak and Negami, is widely used thanks to its suitability for mathematical processing. In this equation, $M_{\mathrm{S}}$ and $M_{\infty}$ are the dielectric modulus on the low- and high-frequency sides of the relaxation, where $\tau$ is the central relaxation time and $\omega$ is the radial frequency; $\alpha$ and $\beta$ are fractional shape parameters describing the skewing and broadening of the dielectric function, respectively. Both $\alpha$ and $\beta$ range between 0 and 1 . These coefficients act as the deviation from the Debye equation. In fact, when $\alpha$ and $\beta$ are equal to 1 , this equation reduces to the Debye equation.

To determine the parameters characteristics of the Havriliak-Negami model $\left(\alpha, \beta, M_{\mathrm{S}}\right.$ and $\left.M_{\infty}\right)$, the experimental $M_{\text {exp }}^{\prime}$ and $M_{\exp }^{\prime \prime}$ data were smoothed through a numerical simulation in the complex plane. The purpose of such a simulation was to find the theoretical values ( $M_{\mathrm{th}}^{\prime}$ and $\left.M_{\mathrm{th}}^{\prime \prime}\right)$. The values of $\alpha, \beta, M_{\mathrm{S}}$ and $M_{\infty}$ that best smoothed the Havriliak-Negami data were obtained by a successive approach method, in which the following expressions 
Table 2. Parameters evaluated by data fitting according to equation (4) for the CWOLP and CWSLP.

\begin{tabular}{ccccccc}
\hline Samples & $T\left({ }^{\circ} \mathrm{C}\right)$ & Relaxation & $\alpha$ & $\beta$ & $M_{\mathrm{S}}$ & $M_{\infty}$ \\
\hline CWOLP & -100 & 1 & 0.70 & 0.89 & 0.159 & 0.234 \\
& -90 & 1 & 0.69 & 0.89 & 0.159 & 0.235 \\
& -80 & 1 & 0.69 & 0.89 & 0.159 & 0.234 \\
& -70 & 1 & 0.69 & 0.89 & 0.159 & 0.234 \\
& -60 & 1 & 0.69 & 0.89 & 0.159 & 0.234 \\
& -50 & 1 & 0.68 & 0.89 & 0.155 & 0.232 \\
& -40 & 1 & 0.75 & 0.89 & 0.154 & 0.229 \\
& -30 & 1 & 0.80 & 0.87 & 0.141 & 0.224 \\
& -20 & 1 & 0.95 & 0.77 & 0.127 & 0.219 \\
& -100 & 1 & 0.70 & 0.85 & 0.154 & 0.217 \\
& -90 & 1 & 0.69 & 0.83 & 0.148 & 0.217 \\
& -80 & 1 & 0.69 & 0.83 & 0.144 & 0.215 \\
& -70 & 1 & 0.69 & 0.80 & 0.140 & 0.215 \\
& -60 & 1 & 0.69 & 0.82 & 0.136 & 0.207 \\
& -50 & 1 & 0.67 & 0.83 & 0.130 & 0.207 \\
& -40 & 1 & 0.73 & 0.84 & 0.126 & 0.205 \\
& -30 & 1 & 0.99 & 0.70 & 0.123 & 0.199 \\
\hline
\end{tabular}

were minimized:

$$
\begin{aligned}
& \chi_{M^{\prime}}^{2}=\sum_{i}\left(M_{\mathrm{th}}^{\prime}-M_{\mathrm{exp}}^{\prime}\right)^{2}, \\
& \chi_{M^{\prime \prime}}^{2}=\sum_{i}\left(M_{\mathrm{th}}^{\prime \prime}-M_{\mathrm{exp}}^{\prime \prime}\right)^{2} .
\end{aligned}
$$

The parameters evaluated by the fitting data are listed in table 2 .

It has been proven that only one-quadruplet value is able to tone with these conditions. The values of $\alpha$ and $\beta$ of the first dielectric relaxation for CWOLP and CWSLP go in the same line with the Havriliak-Negami response. Hence, the structural defects in silica porous ceramics affect the nature of the first dielectric relaxation differently in comparison with those in porous glasses [19].

Figure 6 illustrates the strength evolution of this relaxation in permittivity values, defined by $\Delta \varepsilon=\varepsilon_{\mathrm{S}}-\varepsilon_{\infty}$ [27], vs. the measured temperature for CWOLP and CWSLP. The obtained dielectric strength of CWOLP and CWSLP is close to that of bulk ice [28]. Such a result shows that there is enough amount of water inside the pores for the formation of the ice-like structure for both ceramics. Nevertheless, this dielectric strength exhibits an increase in intensity with temperature for CWSLP and a steadily constant tendency with temperature for CWOLP at the beginning and a sharp increase at the end. Furthermore, the intensity is slightly higher for CWSLP. Therefore, the lateral surface state of the sample affects the intensity and variation with temperature of the first dielectric relaxation strength.

To further probe the water-inner pore surfaces interaction, the second dielectric relaxation was analysed. Figure 7 presents the temperature dependence of its dielectric relaxation time. Its variation reveals non-monotonic relaxation

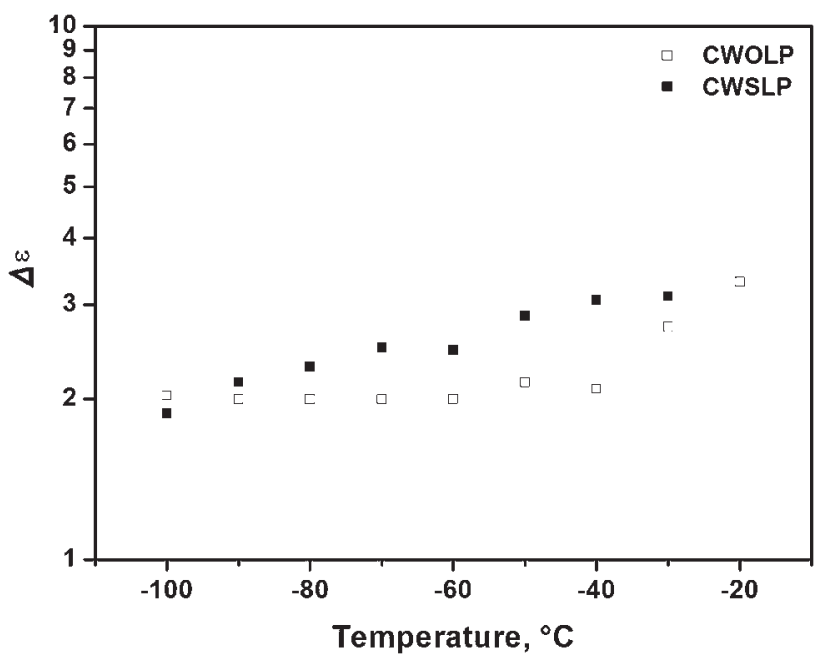

Figure 6. Temperature dependence of the first dielectric relaxation intensity $\Delta \varepsilon$ for (a) CWOLP and (b) CWSLP.

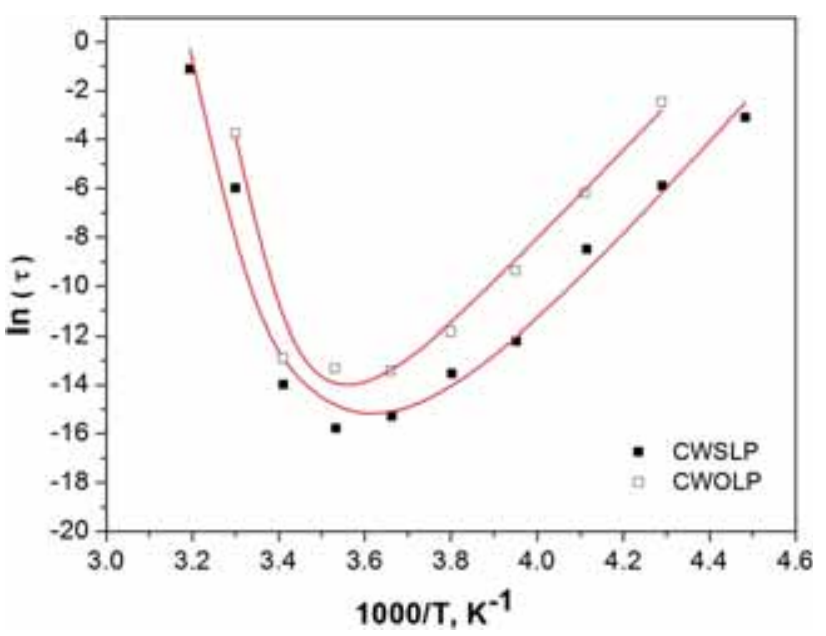

Figure 7. Temperature dependence of the dielectric relaxation time of water confined in CWOLP ( $\square$ ) and CWSLP (ם). Full lines correspond to the best fit according to equation (7). Sample CWOLP: $\ln \tau_{0}=-81.2 \pm 3.8, E_{\mathrm{a}}=151.7 \pm 8.2 \mathrm{~kJ} \mathrm{~mol}^{-1}$, $E_{\mathrm{b}}=70.60 \pm 0.14 \mathrm{~kJ} \mathrm{~mol}^{-1}, C=2.64 \times 10^{13} \pm 0.45 \times 10^{13}$. Sample CWSLP: $\ln \tau_{0}=-91.7 \pm 6.9, E_{\mathrm{a}}=164.9 \pm 14.9 \mathrm{~kJ} \mathrm{~mol}^{-1}$, $E_{\mathrm{b}}=35.19 \pm 0.20 \mathrm{~kJ} \mathrm{~mol}^{-1}, C=2.13 \times 10^{7} \pm 0.71 \times 10^{7}$.

kinetics. The model used in this analysis is based on that introduced to describe the relaxation properties of water adsorbed on the inner surfaces of porous glasses $[11,19]$. According to this model the relaxation time is defined by the following equation [11]:

$$
\ln \left(\frac{\tau}{\tau_{0}}\right)=\frac{E_{\mathrm{a}}}{k_{\mathrm{b}} T}+C \exp \left(-\frac{E_{\mathrm{b}}}{k_{\mathrm{b}} T}\right)
$$

where $E_{\mathrm{a}}$ is the activation energy of a water molecule reorientation, $E_{\mathrm{b}}$ is regarded as the defect formation energy, $k_{\mathrm{b}}$ is the Boltzmann constant and $T$ is temperature. $C=n_{0} v_{0} / v$ is 
a confinement factor, where $v_{0}$ is the volume of a molecule (a mobile unit), $n_{0}$ is regarded as the maximum possible defect concentration and $v$ is the total volume of the system [11].

The fitting curves presented in figure 7 show a good agreement between the experimental and theoretical data given by this model. The fitted values of $E_{\mathrm{a}}$ for both ceramics are higher than the energy attributed to the water molecule reorientation for bulk ice I, which is evaluated as $55.5 \mathrm{~kJ} \mathrm{~mol}^{-1}$ [24,29]. The kinetic transition due to water molecule reorientation in the vicinity of a defect seems to be more difficult, which can be related to a better water-inner surfaces interaction. Indeed, the fitting values of $E_{\mathrm{b}}$ for both ceramics are higher than the energy attributed to the defect formation of bulk ice I, which is evaluated as $32.9 \mathrm{~kJ} \mathrm{~mol}^{-1}$ $[24,29]$. In addition, the comparison of these values revealed that CWOLP shows a better interaction as its energy $E_{\mathrm{b}}$ was the highest [11]. Hence, this quantitative analysis of the kinetic transition process confirmed an enhancement of the water-inner surfaces interaction when the lateral pores of the porous silica ceramic were opened.

\section{Conclusions}

Dielectric measurements were performed on porous silica ceramics to prove the effect of the lateral surface state of the sample on the water-inner surfaces interaction. The analysis of the dielectric relaxation attributed to the reorientation of water molecules in ice-like water cluster structures revealed a similar ice-like structure in both cases and agreement with the Havriliak-Negami model. However, lateral surface state might affect its dielectric relaxation strength, which could be enhanced when lateral pores are sealed. Moreover, the analysis of the non-monotonic relaxation kinetics taking into consideration water confinement in the porous silica ceramics revealed a better water-inner surfaces interaction when lateral pores of the ceramic were opened.

\section{References}

[1] Kurosaki S 1954 J. Chem. Phys. 58320

[2] Pissis P, Laudat J, Daoukaki D and Kyritsis A 1994 J. NonCryst. Solids 171201

[3] Pissis P, Anagnostopoulou-Konsta A, Apekis L, DaoukakiDiamanti D and Christodoulides C 1991 J. Non-Cryst. Solids 1311174
[4] Sakamoto T, Nakamura H and Wada A 1989 J. Phys. Chem. 93 357

[5] Hilfer R 1996 Adv. Chem. Phys. 42299

[6] Huston A L and Justus B L 1995 Opt. Lett. 20952

[7] Avnir D, Gutfraind R and Farin D 1995 In: Bunde A and Halvin $\mathrm{S}$ (eds) Fractals in science (Berlin: Springer) p 229

[8] Shelekhin A B, Pien S and Ma Y H 1995 J. Membr. Sci. 103 39

[9] Schüller J, Richert R and Fisher E W 1995 Phys. Rev. B 52 15232

[10] Arndt M, Stannarius R, Gorbatschow W and Kremer F 1996 Phys. Rev. E 545377

[11] Ryabov Ya, Puzenko A and Feldman Yu 2004 Phys. Rev. B 69 014204

[12] Feldman Y, Puzenko A and Ryabov Y 2006 Fractals, diffusion, and relaxation in disordered complex systems: a special volume of Adv. Chem. Phys. 133 (pt A) 1

[13] www.Filtrosltd.com

[14] Graja F, Duclos A and Depollier C 2013 Proceedings of the 13th international symposium on nondestructive characterization of materials (NDCM-XIII), 20-24 May, Le Mans, France

[15] Niklasson G A 1989 Physica D 38260

[16] Nik1asson G A 1993 J. Phys.: Condens. Matter 54233

[17] Lira-Olivares J, Marcano D, Lavelle C and Sánchez F G 2000 Rev. Latinoam. Metal. Mater. 2068

[18] Puzenko A, Kozolovich N, Gutina A and Feldman Yu 1999 Phys. Rev. B 6014348

[19] Ryabov Y, Gutina A, Arkhipov V and Feldman Y 2001 J. Phys. Chem. B 1051845

[20] Ghallabi Z, Rekik H, Boufi S, Arous M and Kallel A $2010 \mathrm{~J}$. Non-Cryst. Solids 356684

[21] Howard W, Starkweather J R and Avakian P 1992 J. Polym. Sci. B 30637

[22] Tsangaris G M, Psarras G C and Kouloubi N 1998 J. Mater. Sci. 332027

[23] Chan R K, Davidson D W and Whalley E 1965 J. Chem. Phys. 432376

[24] Eisenberg D and Kauzmann W 1969 The structure and properties of water (Oxford: The Clarendon Press) 296

[25] Mijovic J 2003 In Kremer F and Schönhals A (eds) Broadband dielectric spectroscopy (Berlin-Heidelberg: Springer-Verlag) p 349

[26] Richert R 2005 J. Non-Cryst. Solids 3512716

[27] Jin X, Zhang S and Runt J 2002 Polymer 436247

[28] Hasted J B 1973 In Barton C C and La Pointe PR (eds) (London: William Clowes \& Sons Limited)

[29] Petrenko V F and Whitworth R W 1999 Physics of ice (Oxford: Oxford University Press) p 373 